\title{
贫燃 2,5-二甲基呋喃火焰中初级燃烧产物的产生和鉴定
}

童来会 ${ }^{(1)}$, 徐佳 ${ }^{(1)}$, 李哲名 ${ }^{(1)}$, 李善举 ${ }^{(1)}$, 王占东 ${ }^{(2)}$, 金汉峰 $^{(2)}$, 尧命发 ${ }^{(1)}$, 卫立夏 ${ }^{(1 *}$

(1) 天津大学内燃机燃烧学国家重点实验室, 天津 300072;

(2) 中国科学技术大学国家同步辐射实验室, 合肥 230029

*联系人, E-mail: feilix @tju.edu.cn

2012-10-15 收稿, 2012-12-10 接受

国家自然科学基金(51176134)资助

摘要 利用同步辐射真空紫外光电离质谱结合分子束取样技术研究了燃烧当量比为 0.8 的 2,5-二甲基呋喃(DMF)/氧气/氩气低压层流预混火焰中的初级燃烧产物, 得到了 DMF火焰 的光电离质谱和燃烧中间产物的光电离效率曲线, 从光电离效率曲线得到了相应分子/自 由基的电离能, 将实验测量得到的电离能与文献值或者利用量子化学方法计算得到的理

关键词

2,5-二甲基呋喃 低压预混层流火焰 燃烧中间产物 论值比较, 确定了 DMF 火焰中燃烧中间产物的化学结构, 包括尚未报道的 DMF 的氧化产 物和更高级的衍生物, 并根据这些燃烧中间产物的化学结构推断和分析了 DMF 及其初级 燃烧产物的生成与消耗过程.

化石燃料消耗引起的能源枯竭以及环境恶化使 生物质燃料越来越受到重视. 生物质燃料由于具有 可再生, 符合碳中性要求, 运输安全性高等特点, 被 认为是具有巨大潜力的新能源 ${ }^{[1,2]}$. 目前, 一种新型 的生物质燃料—-2,5-二甲基呋喃(DMF)，越来越受 到研究者的重视. 这源于最近 DMF 燃料制备技术上 的一些突破 ${ }^{[3]}$, 这些突破使得直接从自然界含量丰 富的葡萄糖, 纤维素等经过较简单的几步反应可以 得到 DMF. 与广泛应用的乙醇相比, DMF 有着更高 的燃料密度(DMF: $31.5 \mathrm{MJ} \mathrm{L}^{-1}$; 乙醇: $23 \mathrm{MJ} \mathrm{L}^{-1}{ }^{[6]}$ )以 及不溶于水的特性, 具有广阔的应用前景. 在大规模 应用这种燃料之前, 有必要对其燃烧性质进行相关 的实验与理论研究.

对于呋喃类燃料的早期研究主要集中在 DMF 的 热解方面. Grela 等人 ${ }^{[7]}$ 研究了呋喃, 2-甲基呋喃和 DMF 的低压低温 $(1050 \sim 1270 \mathrm{~K})$ 热解. Lifshitz 等 人 $^{[8 \sim 10]}$ 利用反射激波研究了不同温度范围内呋喃 (1050 1460 K), 2-甲基呋喃(1100 1400 K) 和 DMF $(1070 \sim 1370 \mathrm{~K})$ 的热解反应动力学以及部分反应产物
的生成率, 并给出了一个包含 50 种组分和 180 个基 元反应的 DMF 热解反应机理. Friese 等人 ${ }^{[11]}$ 延伸了 Lifshitz 的研究, 利用激波管探究了在 970 1240 K 温 度范围内, 1.6 和 $4.8 \mathrm{bar}$ 两种压力下 $\mathrm{DMF}$ 与 $\mathrm{H}$ 原子 反应的动力学过程, 并结合理论计算得到了 $\mathrm{DMF}+\mathrm{H}$ 的几种不同反应路径及其分支比. Simmie 等人 ${ }^{[12]}$ 通 过理论计算, 讨论了 DMF 的热解过程, 指出了 $\mathrm{DMF}$ 热解过程中可能产生的一些热解产物, 包括 2,5-二亚 甲基-2,5-二氢呋喃等. 吴学松等人 ${ }^{[13]}$ 研究了 DMF 的 低压预混层流火焰, 并提出了 DMF 的消耗过程. 他 们没有观察到 DMF 的氧化产物, 包括 5-甲基-2-呋喃 甲醛. 这使得他们的实验更像是热解过程.

近期关于 DMF 的燃烧基础研究以及在发动机上 的研究也有了一些报道. Wu 等人 ${ }^{[14 ~ 17]}$ 利用定容燃烧 弹和高速纹影法, 系统地研究了不同燃空当量比, 不 同初始压力和不同温度下 DMF-空气-稀释气的层流 传播和燃烧特性, 获得了拉伸和无拉伸火焰传播速 率, 无拉伸层流燃烧速率和马克斯坦长度, 分析了火 焰的不稳定性. 针对呋喃类燃料在压燃发动机上的 
应用, Wei 等人 ${ }^{[18]}$ 在激波管上利用反射激波方法, 开 展了呋喃/氧气/前气在在温度为 $1320 \sim 1880 \mathrm{~K}$ 不同初 始压力条件下着火滞燃期研究. 分别测量了呋喃燃 料在稀燃 $(\phi=2)$, 化学当量比 $(\phi=1)$ 和贫燃 $(\phi=0.5)$ 条件 下的着火滞燃期. 阐述了当量比, 温度和初始压力对 呋喃滞燃期的影响, 研究了呋喃的着火特性以及初 级反应路径. 田国弘等人 ${ }^{[19]}$ 率先在单缸直喷点火发 动机上进行了实验，对比了 DMF，乙醇和汽油的燃 烧与排放情况, 结果表明 DMF 相比乙醇具有一定的 优势. Daniel 等人 ${ }^{[20]}$ 在同一发动机上比较了不同点火 时刻和不同负荷下这 3 种燃料的燃烧排放特性，其中 指出 DMF 的爆震极限没有乙醇高, 这一现象与 DMF 的高辛烷值不相符. 由于尚无具体的 DMF 的燃烧机 理, 他们的结果很少涉及 DMF 的化学燃烧过程, 难 以对某些实验现象进行合理解释.

在实际的发动机中, 进气充量难以实现绝对的 均质，缸内混合气存在着局部过浓和过稀区域. 为了 研究过稀区域的燃料消耗路径以及最终的燃烧产物, 本文利用同步辐射真空紫外光电离质谱结合分子束 取样技术研究了燃烧当量比 $(\phi)$ 为 0.8 的 $\mathrm{DMF} /$ 氧气/ 氩气低压预混层流火焰, 通过理论分析确定了 DMF 火焰中初级燃烧产物的化学结构. 这些初级燃烧产 物通过在燃烧过程中通过燃料的初级反应得到, 其 中包括尚未报道的 DMF 的氧化产物和更高级的衍生 物, 并根据这些燃烧中间产物的化学结构推断和分 析了 DMF 及其初级燃烧物的生成与消耗过程. 这为 DMF 的燃烧机理的构建提供了实验数据.

\section{1 实验装置和方法}

实验在中国科学技术大学国家同步辐射实验室 燃烧与火焰实验站上完成. 实验装置的详细介绍参 见文献[21]. 实验装置主要由燃烧室, 差分抽气室和 光电离室三部分组成. 燃烧室包括进样系统、燃烧炉、 压力监测和控制系统. 利用质量流量计(Multi Gas Controller 647C, MKS, 美国)精确控制气体流量, 微 进样注射泵(ISCO 公司, 美国)精确控制液体燃料流 量. 燃料在燃烧室形成一低压层流火焰. 差分抽气室 包括特制的石英喷嘴和真空系统. 利用特制的石英 喷嘴在火焰中取样. 燃烧产物经过石英喷嘴取样后 形成超声分子束, 分子束经过镍制漏勺准直后进人 光电离室. 光电离室包括一套反射式飞行时间质谱 仪和真空系统. 经过准直后的样品束在光电离室与
同步辐射光垂直相交并被电离，产生的离子利用反 射式飞行时间质谱仪探测, 并由计算机记录.

实验中, $\mathrm{DMF}(\geqslant 99 \%$, 北京利源新赛化工有限公 司)流量为 $0.737 \mathrm{~mL} \mathrm{~min}$, $^{-1}$, 氧气和氩气流量分别为 1.456 和 $1.388 \mathrm{~L} \mathrm{~min}^{-1}$, 燃烧室压力为 30 Torr (4.0 $\mathrm{kPa})$. DMF 在 $150^{\circ} \mathrm{C}$ 汽化后与氧气和氩气混合进人燃 烧炉，利用铇丝点火. 待实验条件稳定后即可得到低 压层流预混火焰.

实验中通过扫描光子的能量, 测量不同光子能 量下的光电离质谱. 得到离子信号强度随光子能量 的变化曲线, 即光电离效率曲线(PIE). 从 PIE 可以得 到相应物种的电离能. 将实验测量得到的电离能与 相应质荷比 $(m / z)$ 的燃烧中间产物的电离能的文献值 或计算值相比较，即可确定燃烧中间产物的化学结 构. 实验测得的电离能由于信号强度不同, 误差一般 为 $0.05 \sim 0.10 \mathrm{eV}$.

部分燃烧中间产物的电离能通过量子化学方法 在 $\mathrm{G} 3 \mathrm{~B} 3$ 水平上得到. 计算电离能的误差为 $\pm 0.15 \mathrm{eV}$.

\section{2 结果和讨论}

\section{1 光电离质谱}

图 1 所示为燃烧当量比为 0.8 , 不同取样位置处的 DMF 的火焰的光电离质谱. 此时光子能量为 $11.00 \mathrm{eV}$, 取样位置为采样喷嘴与燃烧炉表面的距离. 从图 1 中 可以观察到许多质谱峰，其中最强信号为 $\mathrm{m} / \mathrm{z}=96$, 这主要由燃料分子产生. 其余信号主要由燃烧过程 中产生的燃烧中间产物引起. 从图中可以看到，随着 取样位置的增大, DMF 的离子信号强度逐渐减弱, 到

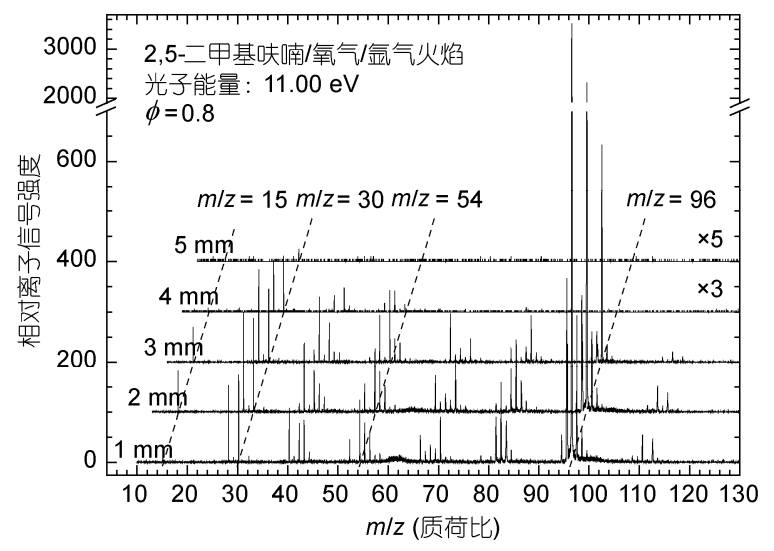

图 1 不同取样位置处 2,5-二甲基呋喃火焰的光电离质谱 燃烧当量比 0.8 ; 光子能量 $11.00 \mathrm{eV}$ 
$4.0 \mathrm{~mm}$ 时已观察不到 DMF 的离子信号, 表明在此位 置时 DMF 已基本消耗完. 与此同时, 其他燃烧中间 产物的离子信号强度先增大后又逐渐减小, 这反映 了这些燃烧中间物种在火焰中先产生，后又被消耗 掉的过程.

\subsection{DMF 的消耗}

图 2(a)所示为在 G3B3 水平上计算的 DMF 的结 构及部分化学键的键长和键能. 从图中可以看到, 甲 基上的 $\mathrm{C}-\mathrm{H}$ 键的键能 $\left(357.4 \mathrm{~kJ} \mathrm{~mol}^{-1}\right.$ ) 比呋喃环上的 $\mathrm{C}-\mathrm{H}$ 键 (496.3 $\mathrm{kJ} \mathrm{mol}^{-1}$ ) 以及 $\mathrm{C}(2)-\mathrm{C}(6)$ 键 $(466.1 \mathrm{~kJ}$ $\mathrm{mol}^{-1}$ ) 的键能低得多, 这意味着 DMF 容易因为这个 甲基 $\mathrm{C}-\mathrm{H}$ 键的断裂而被消耗.

（i ）2-(5-甲基)呋喃基甲基的生成. DMF 可以 通过发生夺氢反应生成 2-(5-甲基)呋喃基甲基 $\left(\mathrm{CH}_{3} \mathrm{C}_{4} \mathrm{H}_{2} \mathrm{OCH}_{2}, m / z=95\right)$ 而被消耗,<smiles>[1H][C@@H](C=CC)c1ccc(C)o1</smiles>

(1)式中 $\mathrm{R}$ 为 $\mathrm{OH}, \mathrm{H}, \mathrm{CH}_{3}$ 或 $\mathrm{O}_{2}$ 等; $\mathrm{HR}$ 相应为 $\mathrm{H}_{2} \mathrm{O}, \mathrm{H}_{2}$, $\mathrm{CH}_{4}$ 或 $\mathrm{HO}_{2}$ 等. DMF 火焰中 $m / z=95$ 的燃烧中间产物 的光电离效率曲线如图 3(a)所示. 在光子能量达到 $6.74 \mathrm{eV}$ 时, 离子信号开始上升, 这意味着光子能量 达到 $6.74 \mathrm{eV}$ 时, $m / z=95$ 的燃烧中间产物开始电离出 现离子信号，这个能量对应的就是该燃烧中间产物 的电离能. 这个能量与 2-(5-甲基)呋喃基甲基的电离 能的计算值 $(6.66 \mathrm{eV})$ 相吻合, 也与吴学松等人 ${ }^{[13]}$ 测 得的富燃火焰中的电离能, $6.63 \mathrm{eV}$ 相吻合. 因此, 这 一燃烧中间产物为 2-(5-甲基)呋喃基甲基. 通过对比 可以发现，贫燃火焰中 2-(5-甲基)呋喃基甲基的电离 能测量值 $(6.74 \mathrm{eV})$ 比富燃火焰中的电离能测量值 $(6.63 \mathrm{eV})$ 偏高, 这是因为在贫燃火焰中燃料浓度较
低, 产生的自由基相对较少, 因此贫燃火焰中 2-(5甲基)呋喃基甲基的信号较弱，测得的电离能误差相 对较大.

如图 2(b)和(c)所示，2-(5-甲基)呋喃基甲基与苯 甲基 $\left(\mathrm{C}_{6} \mathrm{H}_{5} \mathrm{CH}_{2}\right)$ 的结构相似，都是亚甲基直接与芳香 环相连接, 具有烯丙基式的稳定结构. 这样的自由基 反应也相似。

（ii ）2-(5-甲基)呋喃基甲基的氧化．苯甲基可以 通过氧化反应生成苯甲醛 $\left(\mathrm{C}_{6} \mathrm{H}_{5} \mathrm{CHO}\right)^{[22,23]}$,

$$
\mathrm{C}_{6} \mathrm{H}_{5} \mathrm{CH}_{2}+\mathrm{O}_{2} \rightarrow \mathrm{C}_{6} \mathrm{H}_{5} \mathrm{CHO}+\mathrm{OH},
$$

则 2-(5-甲基)呋喃基甲基可通过类似反应氧化成为 5甲基-2-甲酰基呋喃 $\left(\mathrm{CH}_{3} \mathrm{C}_{4} \mathrm{H}_{2} \mathrm{OCHO}, \mathrm{m} / \mathrm{z}=110\right)$,

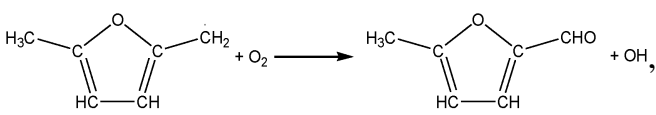

DMF 火焰中 $m / z=110$ 的燃烧中间产物的光电离效率 曲线如图 3(b)所示. 在这个光电离效率曲线上可以观 察到两个电离能, $7.89 \mathrm{eV}$ 和 $8.91 \mathrm{eV}$. 较高的这个电 离能和 5-甲基-2-甲酰基呋喃的电离能的计算值, 8.84 $\mathrm{eV}$ 相吻合. 这说明(3a)式所示反应是可能的. $\mathrm{Wu}$ 等 人 ${ }^{[24]}$ 没有观测到 5-甲基-2-甲酰基呋喃，这可能与他 们所研究的 DMF 火焰是富燃料火焰 $(\phi=2.0)$ 有关. 在富燃料火焰中，氧化反应被抑制，相应的燃烧中间 产物不易被观测到. 5-甲基-2-甲酰基呋喃可以通过醛 基上的夺氢反应被消耗掉,

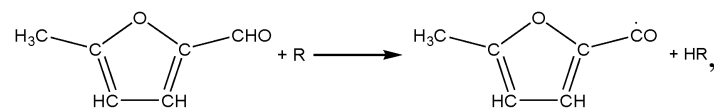

生成的 2-(5-甲基)呋喃基甲酰基 $\left(\mathrm{CH}_{3} \mathrm{C}_{4} \mathrm{H}_{2} \mathrm{OCO}\right.$ )不稳 定，通过脱去一个一氧化碳分子转化成 2-(5-甲基)呋 喃基,

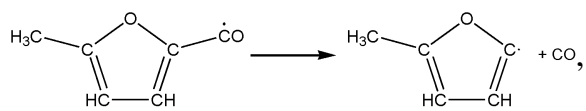

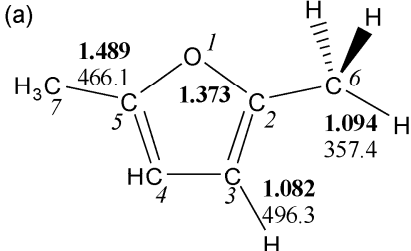

(b)



(c)<smiles>Cc1ccccc1</smiles>

图 2 2,5-二甲基呋喃, 2-(5-甲基)呋喃基甲基和苯甲基的结构

（a）2,5-二甲基呋喃; (b) 2-(5-甲基)呋喃基甲基; (c) 苯甲基. 黑体数字为键长，单位： $\AA$; 斜体数字为键能，单位: $\mathrm{kJ} \mathrm{mol}^{-1}$. 原子周围的斜体序号为原子编号 

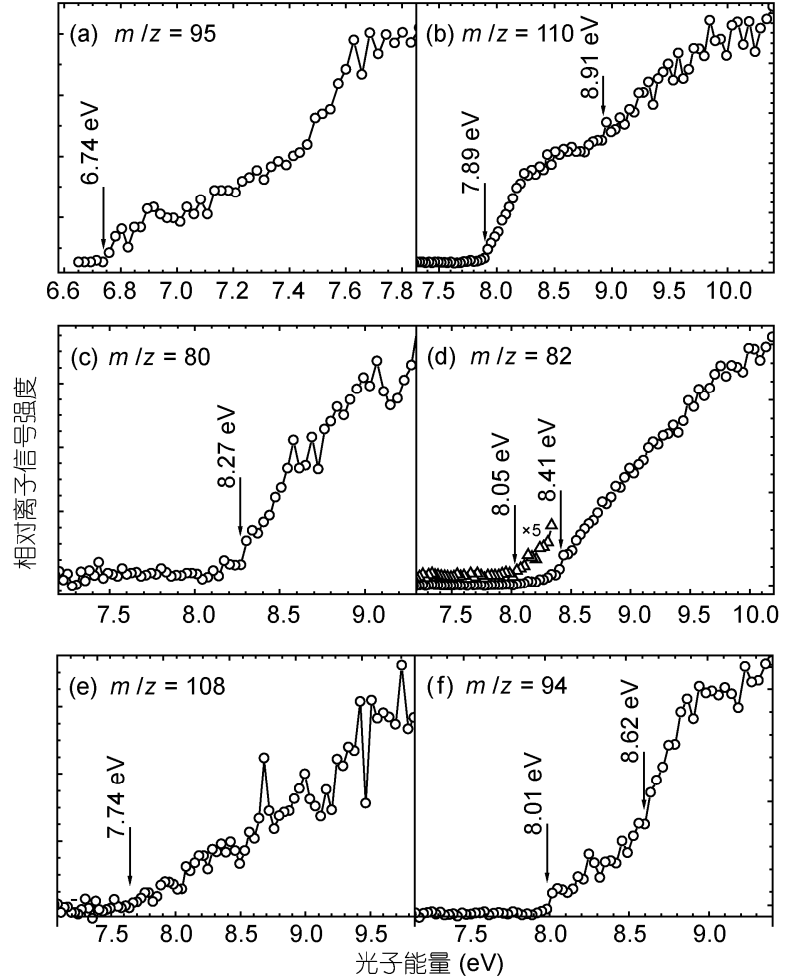

图 32 2,5-二甲基呋喃火焰中部分燃烧中间产物的光电离 效率曲线

(a) $\mathrm{m} / \mathrm{z}=95$; (b) $\mathrm{m} / \mathrm{z}=110$; (c) $\mathrm{m} / \mathrm{z}=80$; (d) $\mathrm{m} / \mathrm{z}=82$; (e) $\mathrm{m} / \mathrm{z}=108$;

(f) $m / z=94$; 燃烧当量比 0.8 , 取样位置 $2.5 \mathrm{~mm}$

这个自由基同样不稳定, 通过呋喃环的开环反应转化 成火焰中常见的丙炔 $\left(\mathrm{HCCCH}_{3}\right)$ 和乙烯酮基( $\left.\mathrm{HCCO}\right)$,<smiles>Cc1ccc(C#CCC=C=O)o1</smiles>

5-甲基-2-甲酰基呋喃也可以通过甲基上的夺氢反应 被消耗掉,<smiles>COc1ccc(CCOc2ccc(C)o2)o1</smiles>

生成的 2-(5-甲酰基)呋喃基甲基通过呋喃环的开环反 应转化成 1 -氧代 - 1,3,4-戊三烯 $\left(\mathrm{CH}_{2}=\mathrm{C}=\mathrm{CH}-\mathrm{CH}=\right.$ $\mathrm{C}=\mathrm{O}, \mathrm{m} / \mathrm{z}=80$ ) 和甲酰基 $(\mathrm{CHO})$,<smiles>C=C=CC=CCOc1ccc(C)o1</smiles>

顺式 1-氧代-1,3,4-戊三烯的计算电离能为 $8.19 \mathrm{eV}$, 这个计算值与 DMF 火焰中 $m / z=80$ 的燃烧中间产物
的实验电离能, $8.27 \mathrm{eV}$ (如图 3(c)所示)吻合. 此外, 这一燃烧中间产物在 2-甲基呋喃火焰中也观测到 $了^{[25]}$.

除了氧化生成苯甲醛, 苯甲基还可以转化成苯 氧基和甲醛 ${ }^{[21]}$,

$$
\mathrm{C}_{6} \mathrm{H}_{5} \mathrm{CH}_{2}+\mathrm{O}_{2} \longrightarrow \mathrm{C}_{6} \mathrm{H}_{5} \mathrm{O}+\mathrm{CH}_{2} \mathrm{O} \text {. }
$$

这一反应与(2a)式所示反应相竞争. 类似地, 2-(5-甲 基)呋喃基甲基的氧化反应也可以生成 2-(5-甲基)呋 喃基氧基和甲醛,<smiles>COc1ccc(COCCOc2ccc(C)o2)o1</smiles>

2-(5-甲基)呋喃基氧基可能通过呋喃环的开环转化成 反式 $1,4-$ 二氧代-1,3-丁二烯 $(\mathrm{O}=\mathrm{C}=\mathrm{CH}-\mathrm{CH}=\mathrm{C}=\mathrm{O}, \mathrm{m} / \mathrm{z}$ $=82)^{[12]}$,<smiles>COc1ccc(C)o1</smiles>

反式 1,4-二氧代-1,3-丁二烯的计算电离能为 $8.01 \mathrm{eV}$. 如图 3(d) 所示, 在 DMF 火焰中 $m / z=82$ 的燃烧中间 产物的光电离效率曲线上可以观察到两个电离能, $8.05 \mathrm{eV}$ 和 $8.41 \mathrm{eV}$. 反式 1,4-二氧代-1,3-丁二烯的电 离能的计算值与这个较低的实验电离能吻合得很好. 因此, (8)式所示反应也是可能的. 这一燃烧中间产物 在 2-甲基呋喃火焰中并未观测到, 可能是由于在 2甲基呋喃火焰中, 燃料的信号太强, 压制了这个燃烧 中间产物的信号. 而在 DMF 火焰中, 2-甲基呋喃的信 号大大减弱, 使得反式 1,4-二氧代-1,3-丁二烯的信号 比较明显.

(iii）2-(5-甲基)呋喃基甲基与甲基的复合. 与苯 甲基类似，2-(5-甲基)呋喃基甲基也可以与甲基复合 生成 2-乙基-5-甲基呋喃 $\left(\mathrm{CH}_{3} \mathrm{C}_{4} \mathrm{H}_{2} \mathrm{OCH}_{2} \mathrm{CH}_{3}, \mathrm{~m} / \mathrm{z}=\right.$ 110),<smiles>CCCCCCCCCCc1ccc(CCCC)o1</smiles>

2-乙基-5-甲基呋喃的计算电离能为 $7.95 \mathrm{eV}$. 这个计 算值与 DMF 火焰中 $m / z=110$ 的燃烧中间产物的光 电离效率曲线上观察到的较高的电离能, $7.89 \mathrm{eV}$ (如 图 3(b)所示), 吻合得很好. 因此, (9)式所示的反应是 可能的. 2-乙基-5-甲基呋喃在 $\mathrm{Wu}$ 等人 ${ }^{[24]}$ 的研究中未 
见报道.

2-乙基-5-甲基呋喃通过夺氢反应被消耗. 通过 乙基上的夺氢反应, 2-乙基-5-甲基呋喃可以转化成 2乙烯基-5-甲基呋喃 $\left(\mathrm{CH}_{3} \mathrm{C}_{4} \mathrm{H}_{2} \mathrm{OCH}=\mathrm{CH}_{2}, \mathrm{~m} / z=108\right)$,

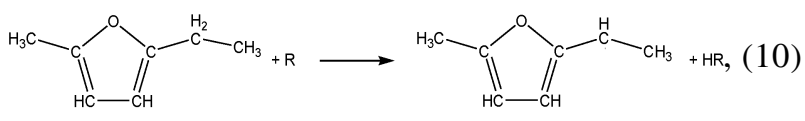<smiles>CC=Cc1ccc(CCCCCc2ccc(C)o2)o1</smiles>

顺式 2-乙烯基-5-甲基呋喃的计算电离能为 $7.79 \mathrm{eV}$. 这个计算值与 DMF 火焰中 $m / z=108$ 的燃烧中间产 物的实验电离能, $7.74 \mathrm{eV}$ (如图 3(e)所示)吻合得很好. 因此, (10)和(11)式所示的反应也是可能的.

（iv）2-(5-甲基)呋喃基甲基的开环反应. 如吴 学松等人 ${ }^{[13,24]}$ 所述, 2-(5-甲基)呋喃基甲基还可以通 过呋喃环的开环反应转化为 1 -氧代-1,3,4-戊三烯和 甲基,

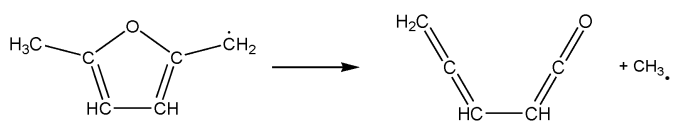

(7)和(12)式所示反应都能生成 1-氧代-1,3,4-戊三烯. 在 2-甲基呋喃火焰中也观测到了这一燃烧中间产物 的生成 ${ }^{[25]}$,<smiles>C=C=CC=CCc1ccco1</smiles>

(7)和(12)式所示都是上述反应的类似反应, 在 DMF 火焰中同时存在是可能的. 如图 3(c)所示, 在燃烧中 间产物 $m / z=80$ 的 PIE 中, 可以观测到一个非常明显 的电离能, $8.27 \mathrm{eV}$. Wu 等人 ${ }^{[24]}$ 的实验结果中, 这个 燃烧中间产物的电离能也非常明显. 考虑到 $\mathrm{Wu}$ 等 并未在富燃料火焰中观测到 5-甲基-2-甲酰基呋喃, 则 R12 应该是火焰中生成 1-氧代-1,3,4-戊三烯的主要 反应.

（V ）2,5-二亚甲基-2,5-二氢呋喃的反应. 如 Simmie 等人 ${ }^{[12]}$ 所述，2-(5-甲基)呋喃基甲基还可能通 过脱氢反应生成 2,5-二亚甲基-2,5-二氢呋喃 $\left(\mathrm{CH}_{2} \mathrm{C}_{4} \mathrm{H}_{2} \mathrm{OCH}_{2}, m / z=94\right)$,<smiles></smiles>

在 DMF 火焰中 $m / z=94$ 的燃烧中间产物的光电 离效率曲线上可以观测到两个电离能, $8.01 \mathrm{eV}$ 和 $8.62 \mathrm{eV}$, 如图 3(f) 所示. 较高的这个电离能与苯酚电 离能的文献值, $8.49 \mathrm{eV}$ 相吻合. 较低的这个电离能与 2,5-二亚甲基-2,5-二氢呋喃的电离能的计算值, 8.05 $\mathrm{eV}$ 吻合得很好. 因此, (14)式所示的反应是可能的.

2,5-二亚甲基-2,5-二氢呋喃可能通过氧化反应被 消耗掉,

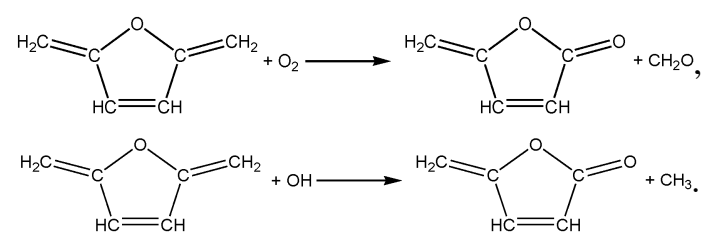

通过氧化反应，2,5-二亚甲基-2,5-二氢呋喃被转 化成 5-亚甲基-2-氧代-2,5-二氢呋喃 $\left(\mathrm{CH}_{2} \mathrm{C}_{4} \mathrm{H}_{2} \mathrm{OO}, \mathrm{m} / \mathrm{z}\right.$ =96). 5-亚甲基-2-氧代-2,5-二氢呋喃的计算电离能 为 $9.41 \mathrm{eV}$. 在 DMF 火焰中 $m / z=96$ 的燃烧中间产物 的光电离效率曲线上观察不到与这个计算电离能相 近的实验值. 这可能是由于燃料分子的信号太强，掩 盖了较弱的产物信号. 此外, 从图 3(f)可以看到, 生 成 5-亚甲基-2-氧代-2,5-二氢呋喃的前体，2,5-二亚甲 基- 2,5-二氢呋喃的信号也比较弱, 这意味着(14)式 所示反应的竞争性可能不如(3(a)), (9)和(12)式强, 因 此生成的氧化产物浓度有限, 以至于观测不到对应 的电离信号.

2,5-二甲基呋喃/氩气/氧气低压预混层流火焰中 观测到的燃烧中间产物如表 1 所示. 2,5-二甲基呋喃 的消耗路径如图 4 所示.

\section{3 总结}

通过 2,5-二甲基呋喃的低压层流预混燃烧研究, 鉴定了火焰中的一系列燃烧中间产物，包括 2,5-二甲 基呋喃的氧化产物和高级衍生物，提出了 2-(5-甲基) 呋喃基甲基的更多的消耗反应，完善了 2,5-二甲基呋 喃的可能的燃烧反应. 2,5-二甲基呋喃主要通过夺氢 反应生成 2-(5-甲基)呋喃基甲基而被消耗; 2-(5-甲基) 呋喃基甲基可以发生氧化反应而被消耗，也可以通 过开环反应而被消耗，还可以与其他自由基反应生 成更高级的呋喃衍生物并进一步被消耗. 此外, 在 2,5-二甲基呋喃火焰中观测到 2-甲基呋喃的生成，因 此, 2,5-二甲基呋喃通过氢原子对甲基的取代反应生 成 2-甲基呋喃也是可能的. 
表 12,5 -二甲基呋喃/氧气/氩气火焰中燃烧中间产物及相应电离能和分子式

\begin{tabular}{|c|c|c|c|}
\hline \multirow{2}{*}{ 质荷比 } & \multicolumn{2}{|c|}{ 电离能 $(\mathrm{eV})$} & \multirow{2}{*}{ 中文名称(分子式) } \\
\hline & 实验值 & 文献值 ${ }^{a)}$ & \\
\hline 15 & 9.80 & 9.84 & 甲基 $\left(\mathrm{CH}_{3}\right)$ \\
\hline 28 & 10.50 & 10.5138 & 乙烯 $\left(\mathrm{C}_{2} \mathrm{H}_{4}\right)$ \\
\hline 30 & 10.83 & 10.88 & 甲醛 $\left(\mathrm{CH}_{2} \mathrm{O}\right)$ \\
\hline 39 & 8.69 & 8.67 & 炔丙基 $\left(\mathrm{C}_{3} \mathrm{H}_{3}\right)$ \\
\hline 40 & 9.71 & 9.692 & 丙二烯 $\left(a \mathrm{C}_{3} \mathrm{H}_{4}\right)$ \\
\hline 40 & 10.34 & 10.36 & 丙炔 $\left(p \mathrm{C}_{3} \mathrm{H}_{4}\right)$ \\
\hline 41 & 8.11 & 8.13 & 烯丙基 $\left(\mathrm{CH}_{2}=\mathrm{CHCH}_{2}\right)$ \\
\hline 42 & 9.57 & 9.617 & 乙烯酮 $\left(\mathrm{CH}_{2} \mathrm{CO}\right)$ \\
\hline 44 & 9.36 & 9.33 & 乙烯醇 $\left(\mathrm{CH}_{2}=\mathrm{CHOH}\right)$ \\
\hline 44 & 10.24 & 10.229 & 乙醛 $\left(\mathrm{CH}_{3} \mathrm{CHO}\right)$ \\
\hline 52 & 9.53 & 9.58 & 乙烯基乙炔 $\left(\mathrm{HCCCH}=\mathrm{CH}_{2}\right)$ \\
\hline 53 & 7.95 & 7.95 & 2-丁炔-1-基 $\left(\mathrm{CH}_{3} \mathrm{CCCH}_{2}\right)$ \\
\hline 53 & 7.95 & 7.97 & 1-丁炔-3-基 $\left(\mathrm{HCCCHCH}_{3}\right)$ \\
\hline 54 & 9.03 & 9.072 & 1,3 -丁二烯 $\left(\mathrm{CH}_{2}=\mathrm{CHCH}=\mathrm{CH}_{2}\right)$ \\
\hline 54 & 9.23 & 9.23 & 1,2 -丁二烯 $\left(\mathrm{CH}_{3} \mathrm{CH}=\mathrm{C}=\mathrm{CH}_{2}\right)$ \\
\hline 55 & 7.49 & 7.49 & 1-丁烯-3-基 $\left(\mathrm{CH}_{3} \mathrm{CHCH}=\mathrm{CH}_{2}\right)$ \\
\hline 56 & 8.87 & 8.95 & 甲基乙烯酮 $\left(\mathrm{CH}_{3} \mathrm{CH}=\mathrm{C}=\mathrm{O}\right)$ \\
\hline 56 & 9.15 & 9.10 & 2-丁烯 $\left(\mathrm{CH}_{3} \mathrm{CH}=\mathrm{CHCH}_{3}\right)$ \\
\hline 66 & 8.54 & 8.57 & 环戊二烯 $\left(c \mathrm{C}_{5} \mathrm{H}_{6}\right)$ \\
\hline 68 & 8.58 & 8.59 & 1,3-戊二烯 $\left(\mathrm{CH}_{3} \mathrm{CH}=\mathrm{CHCH}=\mathrm{CH}_{2}\right)$ \\
\hline 70 & 8.86 & 8.80 & 乙基乙烯酮 $\left(\mathrm{CH}_{3} \mathrm{CH}_{2} \mathrm{CH}=\mathrm{C}=\mathrm{O}\right)$ \\
\hline 70 & 9.62 & 9.65 & 3-丁烯-2-酮 $\left(\mathrm{CH}_{2}=\mathrm{CHCOCH}_{3}\right)$ \\
\hline 78 & 9.23 & 9.24378 & 苯 $\left(\mathrm{C}_{6} \mathrm{H}_{6}\right)$ \\
\hline 80 & 8.27 & $8.19^{\mathrm{b})}$ & 1-氧代-1,3,4-戊三烯 $\left(\mathrm{CH}_{2}=\mathrm{C}=\mathrm{CH}-\mathrm{CH}=\mathrm{C}=\mathrm{O}\right)$ \\
\hline 82 & 8.05 & $8.01^{\mathrm{b})}$ & $1,4-$ 二氧代-1,3-丁二烯 $(\mathrm{O}=\mathrm{C}=\mathrm{CH}-\mathrm{CH}=\mathrm{C}=\mathrm{O})$ \\
\hline 82 & 8.40 & 8.38 & 2-甲基呋喃 $\left(\mathrm{C}_{4} \mathrm{H}_{3} \mathrm{OCH}_{3}\right)$ \\
\hline 94 & 8.01 & $8.05^{\mathrm{b})}$ & 2,5 -二亚甲基-2,5-二氢呋喃 $\left(\mathrm{CH}_{2} \mathrm{C}_{4} \mathrm{H}_{2} \mathrm{OCH}_{2}\right)$ \\
\hline 94 & 8.62 & 8.49 & 苯酚 $\left(\mathrm{C}_{6} \mathrm{H}_{5} \mathrm{OH}\right)$ \\
\hline 95 & 6.74 & $6.66^{\mathrm{b})}$ & 2-(5-甲基)呋喃基甲基 $\left(\mathrm{CH}_{3} \mathrm{C}_{4} \mathrm{H}_{2} \mathrm{OCH}_{2}\right)$ \\
\hline 96 & 7.95 & 8.03 & 2,5-二甲基呋喃 $\left(\mathrm{CH}_{3} \mathrm{C}_{4} \mathrm{H}_{2} \mathrm{OCH}_{3}\right)$ \\
\hline 108 & 7.74 & $7.79^{\mathrm{b})}$ & 2-乙烯基-5-甲基呋喃 $\left(\mathrm{CH}_{3} \mathrm{C}_{4} \mathrm{H}_{2} \mathrm{CH}=\mathrm{CH}_{2}\right)$ \\
\hline 110 & 7.89 & $7.95^{\mathrm{b})}$ & 2-乙基-5-甲基呋喃 $\left(\mathrm{CH}_{3} \mathrm{C}_{4} \mathrm{H}_{2} \mathrm{C}_{2} \mathrm{H}_{5}\right)$ \\
\hline 110 & 8.91 & 8.84 & 5-甲基-2-甲酰基呋喃 $\left(\mathrm{CH}_{3} \mathrm{C}_{4} \mathrm{H}_{2} \mathrm{CHO}\right)$ \\
\hline
\end{tabular}

a) 文献值 ${ }^{[26]}$; b) 计算值 




图 4 2,5-二甲基呋喃的消耗路径

感谢中国科学技术大学国家同步辐射实验室燃烧与火焰实验站齐飞教授对实验工作的支持和指导以及张李东博 士在理论计算方面的建议.

\section{参考文献}

1 Demirbas A. Political, economic and environmental impacts of biofuels: A review. Appl Energy, 2009, 86(Suppl 1): S108-S117

2 Agarwal A K. Biofuels (alcohols and biodiesel) applications as fuels for internal combustion engines. Prog Energy Combust Sci, 2007, 33: 233-271

3 Román-Leshkov Y, Barrett C J, Liu Z Y, et al. Production of dimethylfuran for liquid fuels from biomass-derived carbohydrates. Nature, 2007, 447: 982-985

4 Zhao H B, Holladay J E, Brown H, et al. Metal chlorides in ionic liquid solvents convert sugars to 5-hydroxymethylfurfural. Science, 2007, 316: $1597-1600$

5 Mascal M, Nikitin E B. Direct, high-yield conversion of cellulose into biofuel. Angew Chem Int Ed, 2008, 120: 8042-8044

6 Binder J B, Raines R T. Simple chemical transformation of lignocellulosic biomass into furans for fuels and chemicals. J Am Chem Soc, 2009, 131: 1979-1985

7 Grela M A, Amorebieta V T, Colussi A J. Very low pressure pyrolysis of furan, 2-methytfuran, and 2, 5-dimethylfuran: The stability of the furan ring. J Phys Chem, 1985, 89: 38-41

8 Lifshitz A, Bidani M, Bidani S. Thermal reactions of cyclic ethers at high temperatures 3. pyrolysis of furan behind reflected shocks. J Phys Chem, 1986, 90: 5373-5377 
9 Lifshitz A, Tamburu C, Shashua R. Decomposition of 2-methylfuran: Experimental and modeling study. J Phys Chem A, 1997, 101: 1018-1029

10 Lifshitz A, Tamburu C, Shashua R. Thermal decomposition of 2,5-dimethylfuran: experimental results and computer modeling. J Phys Chem A, 1998, 102: 10655-10670

11 Friese P, Simmie J, Olzmann M. The reaction of 2,5-dimethylfuran with hydrogen atoms-An experimental and theoretical study. Proc Combust Inst, 2013, 34: 233-239

12 Simmie M, Metcalfe K. Ab Initio Study of the Decomposition of 2,5-Dimethylfuran. J Phys Chem A, 2011, 115: 8877-8888

13 吴学松, 卫立夏，黄佐华，等. 2,5-二甲基呋喃/氧气/氩气低压预混层流火焰燃烧中间物种的鉴定. 科学通报, 2008, 53: 2872-2880

14 Wu X S, Huang Z H, Wang X G, et al. Laminar burning velocities and flame instabilities of 2,5-dimethylfuran-air mixtures at elevated pressures. Combust Flame, 2011, 158: 539-546

15 Wu X S, Huang Z H, Jin C, et al. Measurements of laminar burning velocities and Markstein lengths of 2,5-dimethylfuran-air-diluent premixed flames. Energy Fuels, 2009, 23: 4355-4362

16 Wu X, Huang Z, Jin C, et al. Laminar burning velocities and Markstein lengths of 2,5-dimethylfuran-air premixed flames at elevated temperatures. Combust Sci Technol, 2011, 183: 220-237

17 吴学松, 黄佐华, 金春, 等. 2,5-二甲基呋喃-空气混合气层流燃烧速率的测定. 工程热物理学报, 2010, 31: 1073-1076

18 Wei L J, Tang C L, Man X J, et al. High temperature ignition delay times and kinetic study of furan. Energy Fuels, 2012, 26: 2075-2081

19 田国弘, 徐宏明, Danniel R, 等. 2,5-二甲基呋喃的喷雾特性及发动机适应性. 汽车安全与节能学报, 2010, 1: 132-140

20 Daniel R, Tian G H, Xu H M, et al. Effect of spark timing and load on a DISI engine fuelled with 2,5-dimethylfuran. Fuel, 2011, 90: 449-458

21 Qi F, Yang R, Yang B, et al. Isomeric identification of polycyclic aromatic hydrocarbons formed in combustion with tunable vacuum ultraviolet photoionization. Rev Sci Instrum, 2006, 77: 084101

22 Tian Z Y, Pitz W J, Fournet R, et al. A detailed kinetic modelling study of toluene oxidation in a premixed laminar flame. Proc Combust Inst, 2001, 33: 233-241

23 张庆峰, 郑朝蕾, 何祖威, 等. 适用于 HCCI 燃烧研究的甲苯参比燃料化学动力学简化模型. 物理化学学报, 2011, 27: 530-538

24 Wu X S, Huang Z H, Yuan T, et al. Identification of combustion intermediates in a low-pressure premixed laminar 2,5-dimethylfuran/ oxygen/argon flame with tunable synchrotron photoionization. Combust Flame, 2009, 156: 1365-1376

25 尧命发, 李哲明, 童来会, 等. 2-甲基呋喃/氧气/氩气低压预混层流火焰燃烧中间产物的鉴定. 燃烧科学与技术, 2012, 18: 385-392

26 National Institute of Standards and Technology. NIST Chemistry WebBook. http://webbook.nist.gov/chemistry

\title{
Formation and identification of primary combustion derivatives in a lean 2,5-dimethylfuran flame
}

\author{
TONG LaiHui ${ }^{1}$, XU Jia ${ }^{1}$, LI ZheMing ${ }^{1}$, LI ShanJu ${ }^{1}$, WANG ZhanDong ${ }^{2}$, JIN HanFeng ${ }^{2}$, \\ YAO MingFa ${ }^{1} \&$ WEI LiXia ${ }^{1}$ \\ ${ }^{1}$ State Key Laboratory of Engines, Tianjin University, Tianjin 300072, China; \\ ${ }^{2}$ National Synchrotron Radiation Laboratory, University of Science and Technology of China, Hefei 230029, China
}

Low-pressure (4.0 kPa) premixed laminar 2,5-dimethylfuran (DMF)/oxygen/argon flame with an equivalence ratio of 0.8 was studied with tunable vacuum ultraviolet synchrotron radiation photoionization and molecular-beam mass spectrometry. Photoionization mass spectra of $\mathrm{DMF} / \mathrm{O}_{2} / \mathrm{Ar}$ flame and the photoionization efficiency curves of the combustion intermediates were recorded.Ionization energies of the combustion intermediates were obtained from the photoionization efficiency curves. Structures of the combustion intermediates, including the oxidation products and the higher derivatives of DMF, are identified by comparing the measured ionization energies with those reported in literatures or those calculated with quantum chemistry methods. The formation and consumption of DMF and its primary combustion derivatives are proposed based on the combustion intermediates identified.

\section{2,5-Dimethylfuran, low-pressure premixed laminar flame, combustion intermediates}

doi: $10.1360 / 972012-1283$ 